\title{
Skeleton arc additive manufacturing with closed loop control
}

\author{
S.Radel, A.Diourte, F.Soulié, O.Company, C.Bordreuil \\ LMGC and LIRMM- Université de Montpellier - CNRS, Montpellier, France
}

\begin{abstract}
Wire Arc Addtive Manufacturing (WAAM) is a promising direct energy deposition technology to produce high-value material components with a low buy-to-fly ratio. WAAM is able to produce thin-walled structures of large scale and also truss structures without any support. To manufacture complex parts, process reliability and repeatability are still a necessity and this often leads to long developing times. In this paper, a method is proposed to automatically manufacture complex truss structures with point by point arc additive manufacturing and a six axis robot. Computer aided manufacturing (CAM) software is designed to manage (i) material deposition at intersections and (ii) collisions between the part under construction and the torch. Because it is difficult to model the deposition process, the bead geometry is monitored using video imaging. Image treatment program detects the contour of the deposit and computes its current position. With this position, the CAM software corrects the geometry of the part for future deposition. Simple case studies are tested to validate the algorithm. Two solid free form geometries designed by topology optimization are manufactured with this skeleton arc additive manufacturing process.
\end{abstract}

Key words: Welding, Robotics, Closed loop, Truss structure

\section{Introduction}

Wire Arc Additive Manufacturing (WAAM) is a promising directed energy deposition technology. WAAM is able to produce large thin-walled

Email addresses: cyril.bordreuil@umontpellier.fr (S.Radel, A.Diourte, F.Soulié, O.Company, C.Bordreuil) 
structures [1] with an excellent buy-to-fly ratio which is very interesting for high value material. WAAM is a strongly coupled process and is subject to introduce modifications of the bead geometry due to process instabilities or variations. This is one weakness of WAAM. An other area of interest of WAAM is the fabrication of truss structures [2]. The technology is very similar to WAAM but the material is added point by point with a six (or more) axis robot. Different branches of the structure are built by successive metal point addition (and not layer by layer). This additive manufacturing technique enables to follow three dimensional orientations. Topology optimization often leads to difficult-to-manufacture geometries such as those found in nature (tree branches or skeletons for example). These kind of geometries can be produced with this process. This technology will be called Skeleton Arc Additive Manufacturing (SAAM). The final geometry obtained by SAMM has sevral drawbacks. The first is the surface waviness compared to the radius of the branch, the second is access to these branches. These drawbacks will induce extremely difficult machining operations. The technique is therefore more adapted to structures that don't have to be machined. Possible applications are structures with some design constraints or reinforcements in concrete strutures.

Truss structures with fused deposited material were proposed by Mueller et al [3] for fast prototyping. For metal, MX3D has built several parts (bike, bridge,...) [2]. The process is particularly promising for lightweight structures or metallic bio-inspired structures. Compared to rod bending followed by welding, the process is advantageous when structures with multi-curved branches are designed. The robot can generate complex trajectories to follow branches or skeletons. The process can also be combined with other processes such as cutting, milling,etc... The basic technology suffers from some scientific difficulties in managing the deposition of the material for complex geometries.

For the material deposition, Gas Metal Arc Welding is generally the process of choice [1]. This process has inherent fluctuations in the process parameters (wire feed, electric energy) and in the physical properties of the molten zone (surface tension, ....). For SAAM, the material is deposited on a rod and the thermal transfer is very different during the first steps and the last increments of building. The dimensional repeatability during material deposition is a problem that should be solved with on-line control. This problem is not inherent to SAAM. Mazumder et al investigated closed loop during powder deposition. Ding et al [5] proposed in-situ monitoring to con- 
trol laser based direct metal addition moved with an eight axis robot system. Heralic [6] proposed to use 3D scanning to obtain the geometry of the layer and to compensate the difference in height with iterative learning compensation. For WAAM, Xiong et al [9] proposed a closed loop to control variable layer width of thin walled structures based on a charge-coupled device (CCD) camera. In the case of SAAM, direct imaging after the deposition process is a good way to control the shape of the bead.

Numerous works proposed CAM specific for thin-walled components obtained by WAAM. Ding et al [10] developped a method based on $2 \mathrm{~d}$ slices of a CAD file. They developped strategies for crossovers. Their algorithm gave good results for manufacturing thin-walled components. Venturini et al [11] proposed advanced algorithms for deposition tool's path, to manage advanced intersections. For slicing rod geometries, no commercial software is available and two dimensionnal slicer can not be easily extended. The computer aided manufacturing (CAM) software must propagate points along curves with a slicing increment, a starting plane and a building direction. Despite its relative simplicity, two main issues for process planning have to be treated during CAM processing of the wire geometry in order for SAAM to be fully scalable. The first issue is the treatment of intersection of rods. The second is the management of collisions between the torch and the part which is already built. For small contact tip distance to working part and large nozzle diameter, a collision between the nozzle and part under construction can appear at intersection.

The paper presents the setup and the method to circumvent the main issues that can occur during the SAAM fabrication of truss structures with multi-branch and complex geometry. First, a general overview of the setup is given with a description of the material process and the robot. To embed control during processing, a computer must control the whole setup, the different links will be explained. Because SAAM is a new process, only a few articles detail any kind of CAM and no on-line control method was found in the litterature. The next part explains the choice that was made in the CAM to manage intersections and collisions. In the results, the proposed closed loop is tested on academic test studies for validation. To conclude the results part, two multi-branch structures designed by topology optimization are manufactured. In the light of the results, some improvements of the methods are then proposed. 


\section{Overview of SAAM}

The schematic of the SAAM process is shown in figure 1. The main components of the system are: a welding generator to manage the fusion and deposition of the metal, cameras to locate the deposition and a six axis robotic arm to move and orientate the torch .

A personal computer supervises all the steps during the process. A python program on this PC manages data acquisition, CAM, welding and robot instructions. The main inputs for the program are (i) a geometry and (ii) welding parameters. The CAD file can be any format describig wire geometry $($ IGES, STEP,...) or it can be a .stl file that can be skeletonized. After reading this file, the geometry is converted into CAM geometrical entities. The welding parameters can vary with the location of the deposition along the skeleton. A skeleton geometry is shown in figure 1. For this structure, there are many branches with different orientations. The size is more than $300 \mathrm{~mm}$ and can not be manufacture in the power bed of a sintering laser machine.

\subsection{Deposition procedure}

Metal skeleton manufacturing creates similar structures to continuous lattice fabrication [7] or wireprint [3]. In these last techniques, each rod is built without stopping to assure good structural integrity. In SAAM, the building of the different branches is done by increment and is very similar to layer by layer additive manufacturing. The only difference is that the branches are not sliced by a cutting plane but are sliced independently. The slicer detects the first points to build near a starting plane. Then, the algorithm has to propagate points along each branch with an increment $\delta$ corresponding to the height of the deposit.

\subsection{Material and process}

SAAM can be done with any kind of material that can be welded. In this work, a steel grade G3Si was chosen. The base material is a $1 \mathrm{~mm}$ diameter wire. The first points of the structure are generally on a $6 \mathrm{~mm}$ thick steel grade G3Si plate. Some physical properties of the steel are given in table 1. The shielding gas is 92\% Argon and 8\% CO2. The welding power source is a Cold Metal Transfer (CMT) which is a mechanically controlled short-circuit system. CMT is a Gas Metal Arc Welding Process (GMAW) which has the 


\begin{tabular}{|c|c|}
\hline Surface tension in Argon & $1.6 \mathrm{~N} / \mathrm{m}$ \\
\hline Volumetric mass & $7800 \mathrm{~kg} / \mathrm{m}^{3}$ \\
\hline Melt temperature & $1700 \mathrm{~K}$ \\
\hline Thermal diffusivity & $14 e^{-6} \mathrm{~m}^{2} / \mathrm{s}$ \\
\hline
\end{tabular}

Table 1: Some properties of steel G3Si.

advantage to have coaxial energy and mass transfer. The shielding gas is directed with a nozzle of $24 \mathrm{~mm}$ of diameter $\left(\phi_{\text {nozzle }}\right)$.

The CMT process has the advantage (i) to minimize weld pool temperature and (ii) to reduce spatter formation during the transfer. Different process parameters can be controlled such as the wire feed velocity, the welding time, as well as pre and post gas time to protect the weld bead during solidification. All depositions were done with the synergy implemented in the power source. Process settings are often kept constant during the whole process with the exception of the welding time $t_{w}$. This time determines the mass of deposited material and the quantity of energy transfered to the part and is controlled by the supervision software and an external trigger relay. One important parameter to insure a good deposit is the contact tip distance to working part (CTDWP). A CTDWP of around $10 \mathrm{~mm}$ insures a good shielding and a better control of the CMT process. All along the process, CTDWP is kept as constant as possible (see section 3).

In figure 2, a sequence of deposition on a single branch is shown. The physics of deposition can be understood with figure 2. One point is assimilated to a spherical cap with a radius $R_{\text {deposit }}$. Previous studies have shown the complex relationship that can exist between process parameters and the geometry of the spherical cap [8]. The radius $R_{\text {deposit }}$ depends on the quantity of mass and the base radius of the spherical cap. The former is impacted by energetic parameters. If complex curved solid free-form rods need to be manufactured, the base radius of the spherical cap will not be perpendicular to the direction of gravity. Due to gravity, the weld pool can flow and not keep symmetry along the line to be built. Fortunately, one of the main active forces is surface tension that can maintain the drop on the rod. For a drop, the importance of the gravity force relative to the surface tension can be measured with the Bond number:

$$
B o=\frac{\Delta \rho g R_{\text {deposit }}^{2}}{\sigma}
$$


where $\Delta \rho$ is the difference in density between the liquid and the gas (that is negligible here), $\sigma$ is the surface tension and $g$ is the constant of gravity. A Bond number greater than one indicates that gravity has the greatest influence. With the physical properties of table 1, it means that for $R_{\text {deposit }} \leq 3 \mathrm{~mm}$ the surface tension can maintain the melt pool on the rod whatever is the orientation of the deposit relative to the gravity. It means that if the Bond number is then sufficiently low, the weld pool is maintained on the polyline due to surface tension.

To control deposition, the input velocity of the wire or the welding time can be modified. By increasing the wire velocity and maintaining the welding time, the total mass will be higher as well as the total energy transfered to the workpiece, leading to a larger weld pool. Numerous tests were done to find the (wire feed velocity, time of welding) pair for the substrate. Two depositions are shown in figure 3. It is obvious that depending on the value of the couple, the geometry of the depositon will be modified. It was found that wire input velocity of around $2 \mathrm{~m} / \mathrm{min}$ and a welding time of $1.5 \mathrm{~s}$ (without pre and post gas) is a good candidate for deposition with a radius of $3 \mathrm{~mm}$.

In figure 3 , the geometry of the deposit $D_{i}$ is assumed to be a part of a sphere. The main parameters to identify the shape of the deposit are the radius and the center coordinates of the sphere.

\subsection{Six Axis Robot}

In this work, a six axis robotic arm holds the welding torch. A positioning table could be used to add two degrees of freedom, especially for higher radius of rod, to keep the geometry vertical. The robot is controlled with six degrees of freedom $\left(X, Y, Z, R_{X}, R_{Y}, R_{Z}\right)$ in a Cartesian frame. The reproducibility of the positioning is far better that the repeatability of the deposition. The position is given incrementally by the supervision software along the serial port of the robot controller. A library sends the instructions and verifies that it was received (or done) by the robot. Because the part is built point by point, no real-time environment was developed. The work pieces are all manufactured in the center of the workspace of the robot to prevent any kinematic singularities.

A tool coordinate system is located at Tool Center Point (TCP) (see figure 2). The TCP is the point is located to have a CTDWP of $10 \mathrm{~mm}$. In figure 2 , $\left(\vec{x}_{\text {offset }}, \vec{y}_{\text {of fset }}\right)$ corresponds to a frame attached to the workpiece and $\left(\vec{n}_{\text {nozzle }}, \vec{n}_{\text {cam }}\right)$ correponds to the tool frame. 
The main challenge with the industrial robot is to manage its orientation and collisions with the built parts. Collisions will be managed in preprocessing in home-made CAM-software detailed in section 3. The rotations $\left(R_{x}, R_{y}, R_{z}\right)$ are given by the orientation $\vec{n}_{\text {nozzle }}$ of the rod at the current point. A constraint to control the material deposition is that the camera has to be positioned on the positive building direction to prevent any collisions with the built parts. A routine computes the rotations considering that the camera is along the Y-axis of the tool frame $\left(\vec{n}_{\text {cam }}\right.$ in figure 2$)$ and must be finally oriented along $\vec{n}_{\text {cam }}$. By construction, $\vec{n}_{\text {cam }}$ is perpendicular to $\vec{n}_{\text {nozzle }}$ and the scalar product with the building direction $\left(\vec{y}_{\text {offset }}\right.$ in figure 2$)$ is positive.

\subsection{Monitoring}

Detection and control of the deposition is achieved using a CCD camera (640x480 pixels) without filters installed on the side of the welding torch. A chessboard is used to calibrate the camera following the procedure described in [12] to determine distances in the plane of deposit. An image captured by the camera is shown in figure 4(a).

In figure 4(a), the last deposit is on the top and corresponds to the white pixel zone. After the perspective transform in the plane of deposition, the white zone can be used to determine the shape of the last bead and its position relative to the torch. The shape is approximated after several image processing steps: a blur filter is followed by a canny filter, then white pixels are extracted and are chained to form a contour. All the points of the contour are used to fit a circular regression to determine the radius and the center of the deposit. The result is shown in figure 4(b). The spatial resolution can be approximated in figure 4(b) at $30 \mu \mathrm{m}$ for a pixel that is enough compared to 6 $\mathrm{mm}$ of deposit diameter. The region of interest, after calibration, corresponds to a window of 10x15 mm in size in front of the nozzle. Then, because the position of the torch is fixed in the camera frame and after calibration, it is possible to compute the CTDWP in the tool frame.

\subsection{Supervision and overall behavior}

The main program is implemented on a personal computer. The different tasks are ( $i$ ) acquisition of the image for monitoring, (ii) reading of wire frame geometry and slicing it incrementally, (iii) sending instructions to move the six axis robotic arm and (iv) sending a start command to the welding power source. The flow chart of the CAM program is shown in figure 5 . 
The behavior of the supervision is shown in figure 5 . The initial state considers a point $\left(D_{i-1}\right.$ in figure 2$)$ deposited along a polyline and the propagation of it into a point $D_{i}$ (and not yet deposited), this is step (0) in figure 5 . For the point $D_{i}$, the slicer knows the position of the point $\left(x_{i}, y_{i}, z_{i}\right)$ at the top of the deposit and the normal vector $\left(\vec{n}_{\text {branch }}\right)$ to the polyline. Based on possible collision and intersection strategies (see section 3 ), the position and the normal are converted into positions $(X, Y, Z)$ and rotations $\left(R_{x}, R_{y}, R_{z}\right)$ of the robot in the cartesian frame. In the next step (1), the program sends motion instructions in the cartesian robot frame (frame offset in figure 2). In this step, it is very easy to define the approach and release positions and orientations. Once the torch is in position, a relay activates the welding for a duration of $t_{w}$ (step (2)). After this time, the program sends a trigger to the camera to acquire images. Image Processing enables to know where the center of the sphere is located and a new increment is computed, this is step (3). Now, another cycle can be done based on the detection. In the case of figure 2, the center of the sphere is not int its expected position. So the increment $\delta$ that propagates the point is modified in order to have the TCP on the top of the deposit.

\section{Adaptive slicer and control}

\subsection{Geometry}

The wire geometry and its attributes to manage the propagation of the building are detailed in this section. To propagate one point from another on a curve, the simplest way is to define a curvilinear abscissa $s$ on each polyline. Whatever the orientation relative to the building direction, the propagation of a point with an increment $\delta$ simply corresponds to the addition of this increment to the curvilinear abscissa of the current point. For this purpose, the object representing the curve must have a method to give the position and the normal $\vec{n}_{\text {branch }}$ in the world frame for a given curvilinear abscissa. Different types of curve (splines,...) can be used. In this first implementation, a polyline entity is used. A polyline is a list of points that support segments. The curve is limited by a start and an end point. A degree attribute is defined to determine if the point is shared by different polylines. A point with a degree higher than two is a node of the skeleton and is shared by different branches of the skeleton. When the propagation reaches this kind of point, all the polylines that are adjacents to the point are searched. Then, 
building points are then propagated to these adjacent lines except if the polyline is already under construction.

To model the geometry of the construction, every polyline has a deposited radius $R_{d}$ attribute. Based on this radius, a method is implemented on the polyline object to obtain a boundary representation (B-REP) corresponding to a cylinder. This radius is the expected one and is used to predict zones of conflict at convergent intersections or between the rods that were already built and the nozzle (see sections 3.3 and 3.4 ). Two open-source libraries were used to manage the geometry entities depending on their nature: CGAL [13] and OpenCascade [14].

\subsection{Main flow of information}

In this section, step zero of figure 5 is developed. In this step, the new position given by $X^{i}, Y^{i}, Z^{i}$, the rotations $R_{x}^{i}, R_{y}^{i}, R_{z}^{i}$ of the tool and the welding time $t_{w}$ are going to be computed before sending them to the hardware. The multi-branch fabrication is considered so that a list of points to build $l_{\text {pointToBuild }}$, is available based on previous building increments. This list stores different points belonging to different polylines at different curvilinear abscissas. To select the next point to build called $P_{c}$, different criteria can be applied to the points in the list $l_{\text {pointToBuild }}$. The simplest criterion is to choose the point with the smallest distance relative to the starting plane in the direction of building. Other criterion can be added to the position, for example the minimum distance to an axis. As it will be explained in detail in the intersection procedure, the polylines have a priority attribute. Five types of priorities are defined in increasing order: no priority, no conflict priority, low priority, conflict priority and high priority. Depending on this priority, point $P_{p}$ with a priority higher than another point $P_{l}$ can be built before, even if its distance to the base plane is greater than $P_{l}$. The series of actions to slice and control the skeleton from one point to point are:

1. Select point $P_{c}$ in $l_{\text {pointToBuild }}$ and pop it from the list.

2. Compute $t_{w}$ with $P_{c}$ as the center of a sphere of radius $R_{d}$ and intersection with points in $l_{\text {builtPoints }}$.

3. Compute normal $\vec{n}_{n o z z l e}$ depending on intersections.

4. Detect if $P_{c}$ is in a collision zone and which distance $\delta_{\text {coll }}$ is necessary to go out the collision zone.

5. Send instruction to the robot and generator. 
6. Acquire image and detect $x_{c}, y_{c}$ the coordinates of the sphere and $R_{c}$ in the tool frame.

7. With this position and the curvilinear abscissa of $P_{c}$ push a new point in $l_{\text {pointToBuild }}$ and push $P_{c}$ in a list of already deposited points $l_{\text {builtPoints }}$.

Two lists of points are stored throughout the fabrication: $l_{\text {builtPoints }}$ and $l_{\text {pointToBuild }}$. $l_{\text {builtPoints }}$ corresponds to points that were already deposited, $l_{\text {pointToBuild }}$ contains points that were pushed back and that have to be built. Once a point $P_{c}$ is selected from $l_{\text {pointToBuild }}$, welding time is computed. Next the normal is computed. If the point is in a zone with no conflict, the normal is that of the polyline. If the point is in an intersection zone, the orientation is defined to satisfy the strategies given in section 3.4. Before sending space coordinates to the robot, it has to be known if the point is in a zone of possible collision between the tool (the nozzle) and the part under construction (see section 3.3). The point $P_{c}$ is then deposited. An image is taken, an image processing enables to extract the center of the deposit and the radius in the tool frame $\left(\vec{n}_{\text {nozzle }}, \vec{n}_{\text {cam }}\right.$ in figure 2$)$. With the radius, the CTDWP and the curvilinear abscissa of the point of $P_{c}$, the new point can be propagated and pushed to $l_{\text {pointToBuild }}$.

\subsection{Collision procedure}

Different kinds of collision can appear during the additive manufacturing of the skeleton: tool - clamping device, tool - building part... In this part, only the collision between the tool and a polyline of the part under construction is analyzed. The figure 6 shows a collision near a convergent intersection of two branches at different distances from the starting plane. This difference can come from a difference of priority between the two polylines. In function of the difference of priority, considering the line $L_{1}$ was built with $h_{n o z z l e}-h_{\text {deposit }}$ distance in advance relative to $L_{2}$. In figure 6 , the collision occurs between the tool and the building part because the point $\mathrm{C}$ of the nozzle interacts with the deposit. To determine if there is a possible collision, two geometrical conditions have to be checked to know if there is collision. The two conditions are :

$$
\begin{aligned}
& \overrightarrow{C D} \cdot \vec{n}_{\text {nozzle }}>0 \\
& \left(\overrightarrow{C D} \wedge \vec{n}_{\text {nozzle }}\right) \cdot \vec{z}>0
\end{aligned}
$$


where $\vec{y}$ corresponds to the building direction and $\vec{z}$ is normal to $\vec{y}$ and $\vec{n}_{n o z z l e}$. With the geometrical parameters defined in figure 6 , it is straightforward to compute the two criteria and know if there is collision or not. For example for a CTDWP of $10 \mathrm{~mm}, \phi_{\text {buse }}$ of $12 \mathrm{~mm}, h_{\text {deposit }}=h_{\text {nozzle }}=10 \mathrm{~mm}$ and $\theta=45^{\circ}$, the collision is detected. For $\theta=90^{\circ}$, there is always a collision for $h_{\text {depot }}=\phi_{\text {nozzle }} / 2$.

The configuration of figure 6 is for an intersection point but for complex skeletons, collisions can also occur for two rods with a distance lower than $\phi_{\text {nozzle }} / 2+R_{\text {deposit }}$ even if they don't intersect. General cylinder intersection can be complex [15]. Therefore, a more general algorithm based on geometrical parameters of the polyline has to be developed. The collision management is divided into a detection phase and a strategy to solve the collision problem. The purpose of the detection phase is to know if there is an intersection between a cylinder representing the deposit along the polyline and another one representing the envelope of the nozzle along the polyline. With the boundary representations of the cylinders, an intersection procedure is used. The result of the procedure is a list of segments. If there is an intersection, segments are projected on the polyline corresponding to the deposit. The lower and higher curvilinear abscissas of the points projected on one polyline define the zone where a collision can occur. The collision zone must point on the polyline that creates the collision conflict. Each time, a possible collision is found, it is added in a list $l_{\text {collisions }}$. The main advantage of this algorithm is that it can be performed when the geometry is pre-processed. When a point is propagated in a collision zone, a boolean is assigned to the point and a strategy must be put in place. The strategy must satisfy two conditions to safely build a skeleton: (i) a point can not be propagated in the collision zone until the polyline in conflict has not reached its zone of conflict and (ii) the tool can not stay in the collision zone. For this second condition, the tool is always moved at the end of the collision zone with $\delta_{\text {coll }}$. For most collision zones, the CTDWP will be in general higher than the desired one, see section 4.3 .

\subsection{Intersection procedures}

Two strategies must be set depending on the type of intersection relative to the building direction. Divergent and convergent intersections are presented in figure 7 . The type of intersection is detected based on the geometrical characteristics of the two branches. 
For divergent intersections, a high priority is generally defined for one of the two branches. In figure 7(a), the polyline $L_{2}$ was defined with a high priority so that several points of $L_{2}$ are built before starting the deposition of $L_{3}$. If the nozzle is tilted in the direction of $\vec{n}_{L_{3}}$, the welding arc created between the nozzle and the already deposited material on polyline $L_{2}$ can be deviated due to a shortest arc length. This will modify the position of the point of intersection and polyline $L_{3}$ will not be built along the right axis. In order to circumvent this problem, an offset angle is defined $\gamma_{\text {offset }}^{\text {div }}$ to be more perpendicular to the polyline $L_{2}$. This angle is defined in the plane defined by $\left(\vec{n}_{L_{2}}, \vec{n}_{L_{3}}\right)$ and starts along the branch that has priority (polyline $L_{2}$ in figure $\left.7(\mathrm{a})\right)$. If there is no problem for access, this angle is $90^{\circ}$.

For convergent intersections, the strategy is different. In figure 7(b), a convergent intersection is shown. In the pre-processing of the geometry, zones of conflict for deposition at convergent branches are detected in a similar manner to the procedure for collision zones. When a point is propagated inside a zone of conflict, the program changes the priority of rod $L_{1}$ to a conflict priority and this branch is built in priority. The difference in distance priority enables to build the other branch $L_{2}$ of the convergent intersection, but it is not possible to finish the intersection because $L_{2}$ can not be fed by material: $L_{1}$ is built and is an obstacle for the wire to feed branch $L_{2}$. So an offset angle $\gamma_{o f f s e t}^{\text {conv }}$ is defined to rotate the tool around TCP and the direction perpendicular to the plane of $L_{1}$ and $L_{2}$. This angle is set around $70^{\circ}$.

\subsection{Overall algorithm}

The figure 8 shows the overall algorithm including all the procedures. In this part, the link between the different procedures is explained.

The algorithm starts with the detection of all the points closest to the starting plane. These points are added into the list $l_{\text {pointToBuild }}$. Next, a point $P_{c}$ is selected depending on criterion (see section 3.2) in the list $l_{\text {pointToBuild }}$. This point will be used to move the robot. At this point, the direction along the polyline is known : $\vec{n}_{\text {branch }}$. The rules to make strategies for process or deposition are embedded in a object called the manager slicer. The point $P_{c}$ is consdered to be the center of a sphere of radius $R_{d}$ to model geometrically the deposit. The volume of this sphere is noted $V_{b}$. If spheres based on points in the list $l_{\text {builtPoints }}$ and $R_{d}$ have possible intersections with $V_{c}$, the union of sphere $V_{B}$ is built and the volume $V_{c}$ is cut by $V_{B}$. The remaining volume is used to compute the ratio $k_{w}$ of welding time to decrease the quantity of mass. Next, it is searched if the point $P_{c}$ is lying inside a zone of collisions. 
If so, the $\delta_{\text {coll }}$ is used to place the torch outside of this zone. If $\delta_{\text {coll }}$ is too high, the deposition will not be visible in the frame of the CCD camera. In this case, the deposition is done until the deposition can be detected. The collision procedure is done in priority. Next, the manager tests if the point $P_{c}$ lies inside a zone of intersections. If so, the nature of the intersection, convergent or divergent, is determined and the orientation of the nozzle is modified using the previous strategy. Next, the post-processor computes the position and rotation of the robot. This procedure is repeated until the $l_{\text {pointToBuild }}$ is empty.

\section{Results}

\subsection{On line monitoring}

The adaptive slicer enables to propagate points with different increments all along the fabrication. With the supervision, it enables to always place the tool at the same CTDWP. In this part, the on line control is explained on a vertical line. For this single line, no intersection and no collision can occur. The algorithm 8 corresponds to the monitoring phase, the propagation of the point with correction and deposition. In figure 9 , a deposition process $D_{i-1}$ leads to a volume far from the value expected due to thermal conditions, spatters,.... Because deposit $D_{i-1}$ has a radius smaller than the expected one, the distance between the contact tip and the part is higher than 10 $\mathrm{mm}$. If other bad depositions occur, the distance will probably increase and will stop the process because material deposition would not occur where it is wanted. By integrating the monitoring to impose a CTDWP of $10 \mathrm{~mm}$ by controlling the tool position, it is sure that the deposition will occur on work piece.

To monitor the deposition process, a series of images is acquired just after deposition. The first images can not be used due to radiation of the weld pool. The processing to obtain the contour of the deposition is done on the first image that satisfies a criteria on its number of white pixels. The calibration allows to transform the geometry from the camera frame to the tool frame. The top of the deposit is computed. It is then straightforward to compute the new increment to propagate the point. A series of images during the building of a vertical line is shown in figure 10.

In figure 10, the bottom dashed line represents the top of the deposit and the top line corresponds to the position of the contact tip in the tool frame. 
Except for the very first points, the distance is constant for all increments. The control plays its role.

The number of images to reach the white pixels criterion can be related to the solidification time. The study of the time for the images to go beyond the threshold can tell us how the thermal transfer is modified. In this experiment, more than three hundreds successive points were done. In figure 11, the time to reach the criteria is shown in function of the point increment during the manufacturing.

There is a factor ten for the solidification time between the first and the last point. The time increases rapidly for the first points and stabilizes at around twenty seconds at the fiftieth point. The thermal transfer is different at the very first point where the heat goes to the substrate and at the last point where the heat goes to the thin rod and into the air. The second remark goes to the noise on the curve. This is due to fluctuations of energy transfer during the deposition. Some points were detected with lower solidification times, this is in general due to bad mass transfer. This demonstrates the interest of embedding monitoring in this procedure.

\subsection{K skeleton}

The behavior of the strategies proposed for the convergent and divergent intersections are tested on a $\mathrm{K}$ skeleton geometry shown in figure 12 . The purpose is to investigate how the intersection management behaves in a standalone manner and if the desired geometry is correctly approximated. The geometry was chosen to not have any kind of collision between the tool (the torch) and the part. The process parameters are chosen in order to have a deposition with a radius of $3 \mathrm{~mm}$ and a deposit height of $1.3 \mathrm{~mm}$.

Figure 12 shows zones of deposit conflicts that can occur during the manufacturing process. It must be pointed out that this detection is only done once. For this geometry two intersection strategies must be set. The two intersections are based on line $L_{1}, L_{4}$ and $L_{5} . L_{2}$ and $L_{3}$ have a priority in the zone of conflict and $L_{3}$ has a high priority. For the convergent intersection, the offset angle is set to 70 degrees. For the divergent intersection, the offset angle is set to 90 degrees. The welding time is modified with height according to the modification of thermal transfer.

In figure 13, different images acquired by a fixed camera in the world frame are shown for the convergent intersection, for the divergent intersection and the final shape. The additive manufacturing was done automatically without interruption and without any interactions with the software. In figure 13(a), 
a deposition defect can be seen on $L_{1}$. The trouble was detected thanks to the monitoring and the process could continue. Figure 13(a) shows the behavior of the strategy for the convergent intersection. Branch $L_{1}$ is vertical and $L_{4}$ is tilted at thirty degrees. With the image processing, the position of the deposition is known on the polyline and the conflicts are then the same on the product part and in the virtual part in the CAM. The offset angle enables to deposit the material at the intersection. Figure 13(b) presents the deposition at the divergent intersection. It is clear that branch $L_{3}$ was built first. The offset angle leads to the torch being perpendicular to the rod $L_{3}$ (see discussions section for problems). The point is deposited on the border of branch $L_{3}$ so that $\delta$ increment must be different for this kind of intersection compared to classical slicing. In this example, $\delta$ was set at $5.9 \mathrm{~mm}$. Because slicer is adaptive, it is not a major issue to change the increment at intersections. The figure 13(c) shows the free-form at the end of manufacturing. The points and lines on the image are those defined in the CAM software. The lines correspond to neutral axis. There is almost no divergence between the desired geometry (points and lines) and the part that was manufactured. The first look seems to indicate that the manufactured geometry differs from the desired one but the points of intersection of the manufactured part are located at the desired spatial coordinates. The orientations of the branches of the manufactured part are also correctly aligned with those of the CAM geometry.

\subsection{Chair skeleton}

The behavior of the strategy for collision management is tested on a geometry representing a chair. Two branches have a ninety degree angle that will enforce a collision between the nozzle and the deposit. The process parameters remain the same. The deposit radius is still three millimeters and the nozzle radius is around twelve millimeters. In figure 14, the zone of collisions between the tool and the geometry of the work piece are also shown.

It is clear that if branch $L_{2}$ continues to be built while the process is still manufacturing the vertical line of branch $L_{1}$, the tool will be in collision with branch $L_{2}$. The strategy is to wait that the two branches are stopped when points want to be propagated inside the zone of collision. When this condition is met, the two branches are built simultaneously but with a CTDWP higher than $10 \mathrm{~mm}$ in order to locate the tool out of the collision zone. The distance

to be out of the collision zone $\delta_{\text {coll }}$ is used to this purpose. This can lead to 
CTDWP higher than twenty millimeters. In accordance with the algorithm of figure 8, the robot is not moved and there is successive deposits until a weld pool is detected by the camera and then the propagation can occur.

Figure 15 shows a series of images during manufacturing of the chair. Figure 15(a) corresponds to a classical skeleton additive process. Branches $L_{1}$ and $L_{2}$ are built simultaneously. When point on a branch $L_{2}$ enters into the collision zone, the branch is stopped (figure 15(b)) . Then, the branch $L_{1}$ can be built until the point reaches the collision zone (figure 15(c)). Then the two rods are done simultaneously. The figure 15(d) shows the final shape of the chair obtained without any interaction between the CAM and the operator, despite troubleshooting on a deposit on line $L_{1}$. The final geometry is close to the desired one.

\subsection{Cantilever beam skeleton}

Additive manufacturing is promising in particular with its coupling with topology optimization. Shape optimization of elastic structures has become popular. Allaire et al [16] proposed a method based on level-set where the front velocity is derived from the shape sensitivity. They proposed some code to manage plane optimization. This code was used to obtain the geometry of a cantilever beam loaded by a transversal force. The initialization parameters to run the optimization process are the same as the case described in the center of figure 1 of [16]. The geometry is shown in figure 16 .

The structure has several possible collisions zones between the nozzle and the manufactured part. A divergent intersection has to be manufactured in the very first increment. Due to the symmetry of the structure, it is not possible to use an offset angle of 90 degrees, so a angle $\gamma_{o f f s e t}^{\text {div }}$ is chosen at 75 degrees. In the middle of the structure, the branches do not have any intersections and are tangent. Several collisions can occur as can be shown on the right of figure 16. The CAM software that was home-implemented has a virtual mode to observe the behaviour of the different strategies. It was detected that for this structure, the algorithm was not able to detect a collision between an interior branch and an exterior one. To solve this problem, the priority of the line on the border of the structure was considered with no priority in order to first build the point in the collision.

The different images in figure 17 show different increment during fabrication. The required number of points to build the cantilever was around 1200. With the monitoring detecting cooling time, it takes around six hours of fabrication. The last image shows a comparison between the manufactured 
part and the desired geometry implemented in the CAM software. The comparison is excellent and only few differences can be observed. Once again, no manual adjustment was necessary due to the monitoring that enables the knowledge of up-to-date the position of the deposition.

\subsection{Three dimensional skeleton}

The previous example demonstrates the interest and the performance of the method but most results of topology optimization give three dimensional parts. The algorithms and the methodology are tested on a part optimized by F.Mitjana [17]. Details can be found in the thesis.

The final structure was obtained by combining two processes. The two external parts were obtained by plasma cutting. The branches in the middle of the structure were skeletonized to obtain wires. The wires were converted into polylines and then the additive manufacturing was done from one of the two plates. To finish, a welding process was used to join the second plate to the branch. This small example demonstrates the capacity of the SAAM process for optimized structures.

\section{Discussions}

The proposed methodology was presented and was very efficient to manufacture two and three dimensional parts. The most important feature is that the additive manufacturing process is done automatically without any human interactions. The point by point manufacturing is different from the classical WAAM of complex structures but the strategies are very similar. The main weakness of the different examples is the time required to build the structure. Most of the time is spent on the detection of the weld bead. For different purposes, it was chosen to acquire images continuously during solidification. For the vertical building, for high deposit points, it was shown that twenty seconds were necessary before reaching the criterion on white pixels. Another strategy would be to build other points and then to come back for inspection and to evaluate the deposition of the point and to correct the position of the tool. Other types of control can be used to determine CTDWP. For example, it is known that arc voltage during the process is directly linked to the CTDWP. Unfortunately, if there is a trouble during the deposition process, camera is only tool to calculate the quantity of mass that was deposited leading to corrective actions. 
For all the tests, zones of deposition conflicts were preprocessed on the geometry defined in the CAM software. When a conflict near an intersection is detected on this virtual geometry, it has to be located at the same place on the building part. Fortunately, this is the case because the monitoring and the closed loop control the position of the construction on a polyline. For all the geometries defined in this article, it works without any trouble but a good development will be to define a regulation and strategy for the deposition near intersections. It could be particularly interesting for more complex structures such as the cellular lamp of figure 1.

The strategy proposed for divergent intersections (figure $7(\mathrm{a})$ ) can be a problem if two vertical rods are too close and a branch has to start from one of the two vertical branches. It can create a nozzle - building part collision and up to now the intersection and collision procedures are not coupled. For structures with many rods, it is expected to work well because the slicing is done along a building direction and the offset angle is defined to prevent this kind of problem. In figure 17, if a $90^{\circ}$ offset angle was chosen, the nozzle could have a collision with the symmetric branch.

The management of collision proposed in this article is simply based on cylinder intersections. The strategy to stop the two branches works well for an intersection with two rods. For more rods at the same intersection, it can be more complicated and the strategy must be adapted. For example if an intersection corresponding to a trident has to be built, the strategy will be in trouble because the two external branches will be in collision. In this case, it could be interesting to modify the building direction.

All these developments are designed for point wise deposition but the concepts can be adapted to classical WAAM. The main difference is that the deposition process is continuous whereas in the case of this application it is discrete.

To conclude, the method will be fully scalable if larger radius can be deposited. It was discussed that the maximum radius is 3 millimeters due to gravity. To manufacture rods with radius higher than $3 \mathrm{~mm}$ for the G3Si steel, more degrees of freedom have to be used to try to maintain the weld pool horizontal during the manufacturing.

This article gives basic methodology for point wise deposition along branches. Some procedures can be improved, to increase productivity or the complexity of the manufactured part with optimised tool path generation. For our point of view, the main result is the capacity of the proposed algorithm to build part without any human interaction. 
If the obtained geometry is unusual from the mechanical point of view, due to its size and its waviness, it can be interesting in some applications. Combined with thin-walled WAAM structures, it can be a good process to stiffened the structure. If the mechanical behaviour of a part can be modelled, this type of structure can be particulary interesting in energy absorption structure.

\section{Conclusion}

The article has presented a fully automated method to build skeleton free-forms. The originality comes from the coupling of the monitoring of the deposition with the slicing of the skeleton structures to move the robot. All the results were obtained without any human interaction and the results in term of geometry are excellent. Because the manufactured geometry approximated the CAM geometry well, it will be interesting to test the geometry on larger structures such as the cellular lamp of figure 1 . The process is validated and the structural integrity has to be verified by mechanical characterization of the parts that were manufactured.

To improve the SAAM process, different steps can be taken. The first step will be to evolve the control strategy, in order to decrease the manufacturing time. Another development will be to improve the algorithm for collision detection. The algorithm can also use more complex detection procedure. The last modification will be on tool path optimisation. The overall geometry is already modelled as a graph, combined with collision and intersection features and strategies, the tool path could be minimized.

\section{References}

[1] Williams.S.W and Martina.F and Addison.A.C and Ding.J and Pardal.G and Colegrove.P, "Wire + Arc + Additive + Manufacturing", Materials Science and Technology, No 7, p 641-647, Vol32, 2016

[2] MX3D project : http://mx3d.com/

[3] Mueller.S and Im.S and Gurevich.S and Teibrich.A and Pfisterer.L and Guimbretire.F and Baudisch.P, "WirePrint: 3D Printed Previews for Fast Prototyping", UIST14, October 58, p274-280, 2014 
[4] Mazumder.J and Dutta.D and Kikuchi.N and Ghosh.A," Closed loop direct metal deposition: art to part", Optics and Lasers in Engineering, Vol 34, p 397-414, 2000

[5] Ding.Y and Warton.J and Kovacevic.R, "Development of sensing and control system for robotized laser-based direct metal addition system", Additive Manufacturing, Vol 10, p24-35, 2016

[6] Heralic.A and Christiansson.A-K and Lennartson.B, "Height control of laser metal-wire deposition based on iterative learning control and 3D scanning", Optics and Lasers in Engineering, Vol 50, p 12301241, 2012

[7] Eichenhofer.M and Wong.J and Ermanni.P, "Continuous lattice fabrication of ultra-lightweight composite structures", Additive Manufacturing, Vol 18, p 4857, 2017

[8] Chapuis.J and Romero.E and Soulié.F and Bordreuil.C and Fras.G, "Behaviour of spreading molten metal drops deposited by fusion", Experimental Thermal and Fluid Science, Vol 48, Pages 29-36, 2013

[9] Xion.J and Yin.Z and Zhang.W, "Closed-loop control of variable layer width for thin-walled parts in wire and arc additive manufacturing", Journal of Materials Processing and Technology, Vol 233, p 100-106, 2016

[10] Ding.D and Shen.C and Pan.Z and Cuiuri.D and Li.H and Larkin.N and Van duin.S, "Towards an automated robotic arc-welding-based additive manufacturing system from CAD to finished part", Computer Aided Design, Vol 73, p 66-75, 2016

[11] Venturini.G and Montevecchi.F and Bandini.F and Scippa.A and Campatelli.G "Feature based three axes computer aided manufacturing software for wire arc additive manufacturing dedicated to thin walled components", Additive manufacturing, Vol 22, p643-657, 2018

[12] Bradsky.G and Kaehler.A, "Learning OpenCV", O'Reilly Ed, 2008

[13] The CGAL Project, "CGAL User and Reference Manual", ver 4.12, 2018

[14] OpenCascade Project, https://www.opencascade.com/ 
[15] Ketchel.J and Larochelle.P, "Collision Detection of Cylindrical Rigid Bodies for Motion Planning", Proceedings of the 2006 IEEE International Conference on Robotics and Automation Orlando, Florida, p15301535,2006

[16] Allaire.G and Jouve.F and Toader.A-M, "Structural optimization using sensitivity analysis and a level-set method", Journal of computational physics, Vol 194, p363-393, 2001

[17] Mitjana.F, "Optimisation topologique de structures sous contraintes de flambage", Phd Université de Toulouse, 2018 


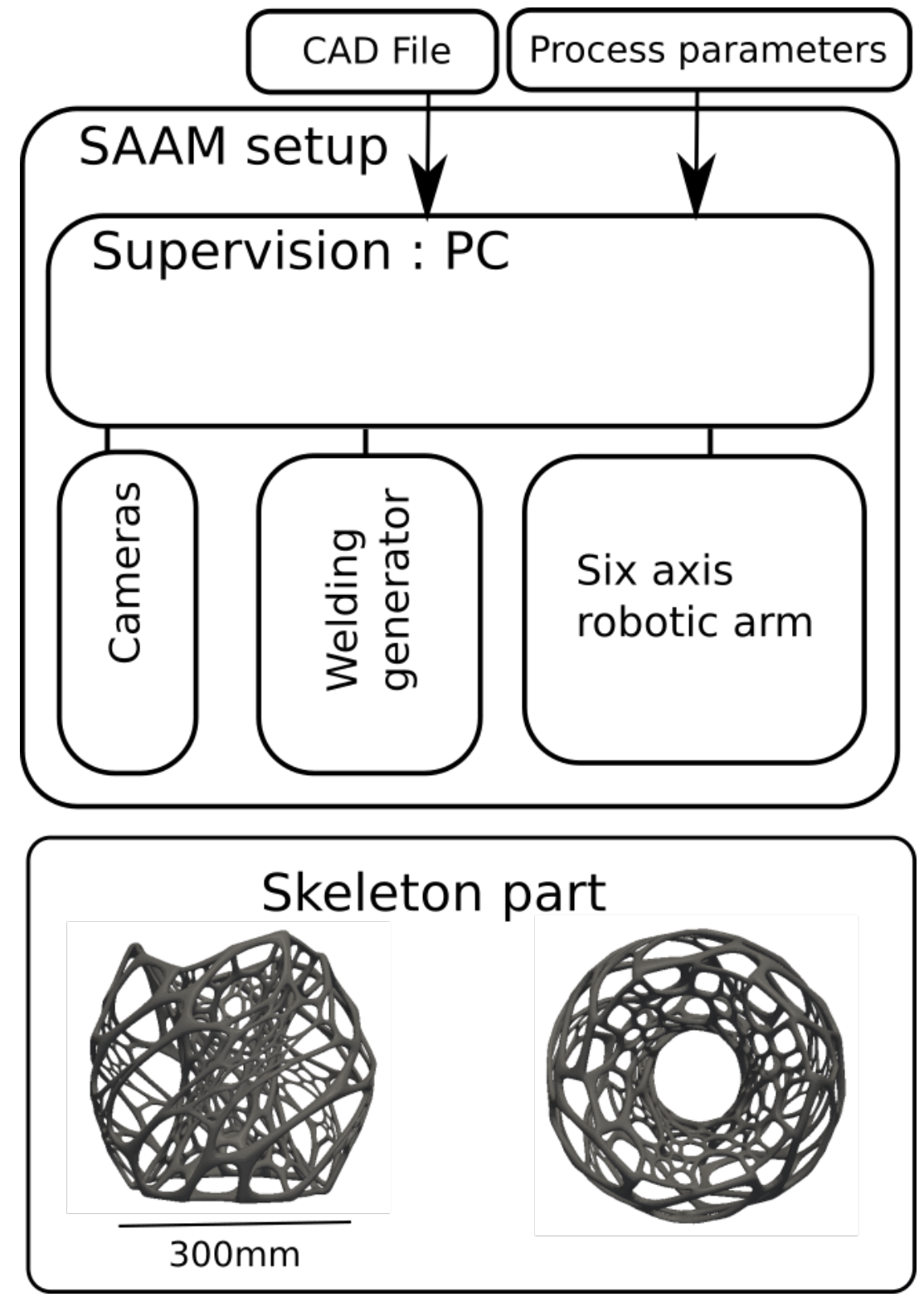

Figure 1: The SAAM setup. All the hardwares are waiting for instructions from the PC. 


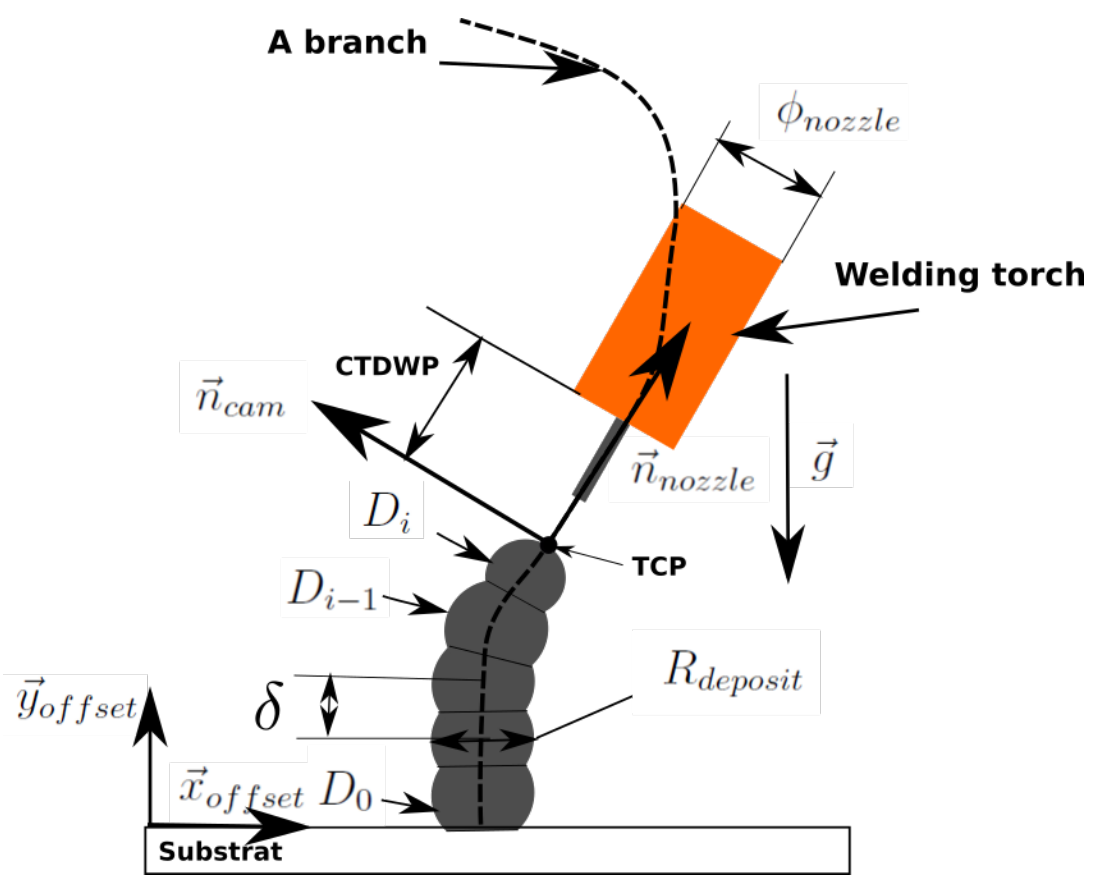

Figure 2: Several depositions $\left(D_{0}, \ldots . ., D_{i-1}\right)$ along a trajectory for a single branch. Troubleshooting occurs during the last deposition $D_{i}$ leading to lower radius than the planned one.
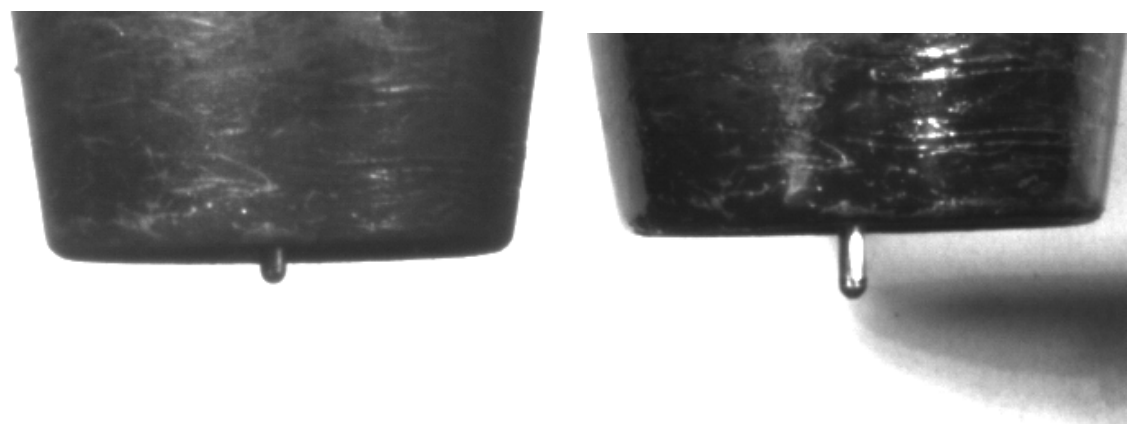

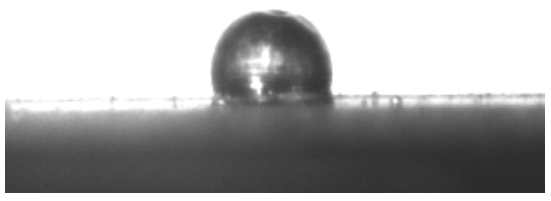

(a)

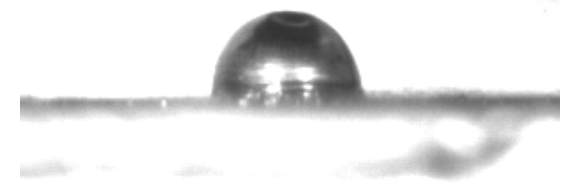

(b)

Figure 3: Depostions obtained with (a) (wire feed velocity:2m/min, $t_{w}=3 s$ ) and (b) $(3 \mathrm{~m} / \mathrm{min}, 2.5 \mathrm{~s})$ 


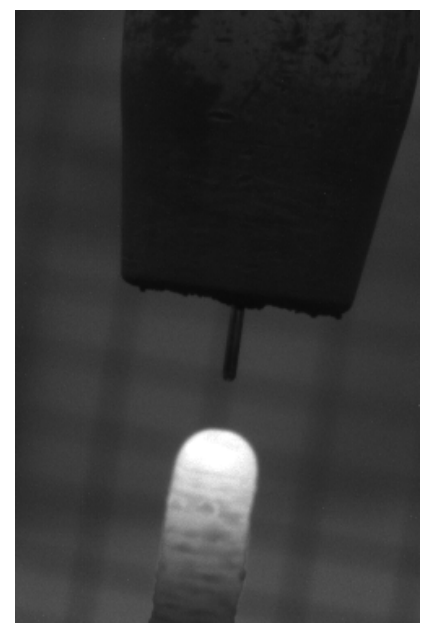

(a)

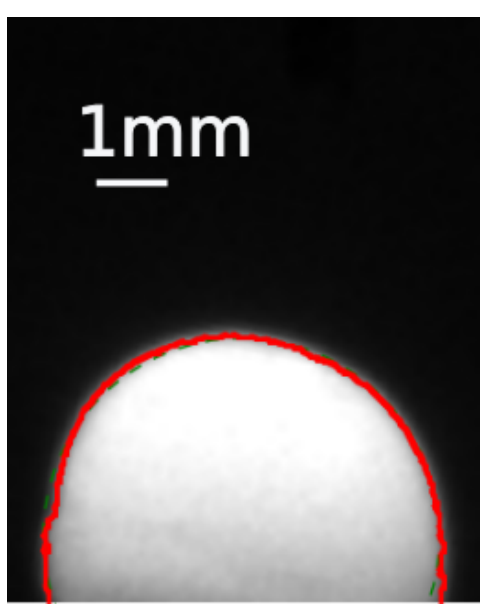

(b)

Figure 4: An image with camera fixed to the torch (a) and the result after calibration and image processing (b).

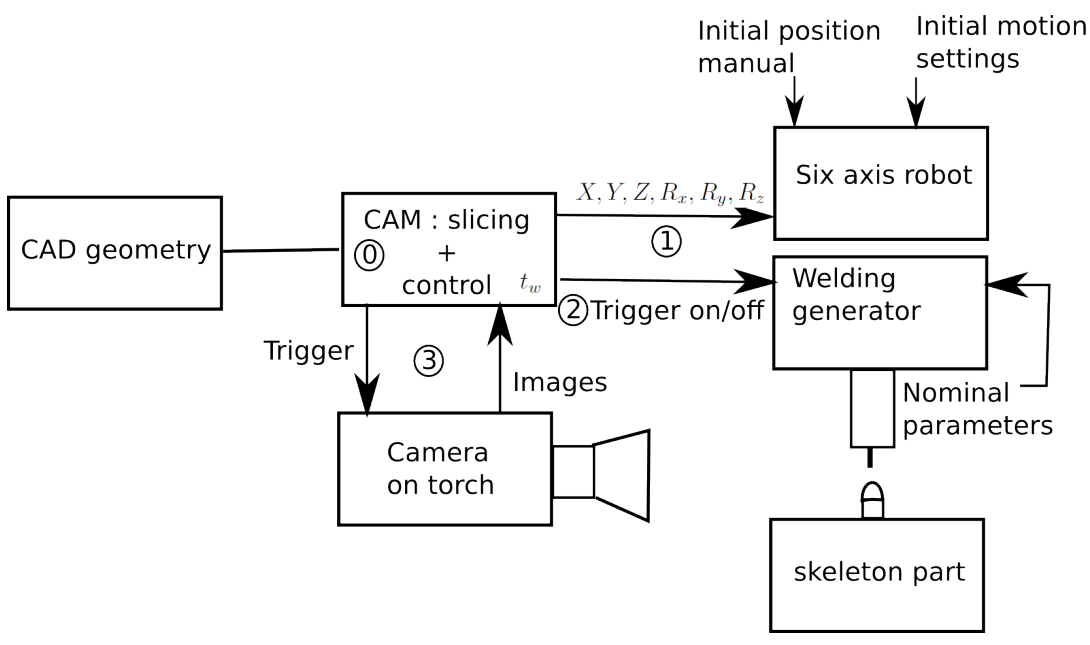

Figure 5: Flow chart of information to manage on line control and slicing. 


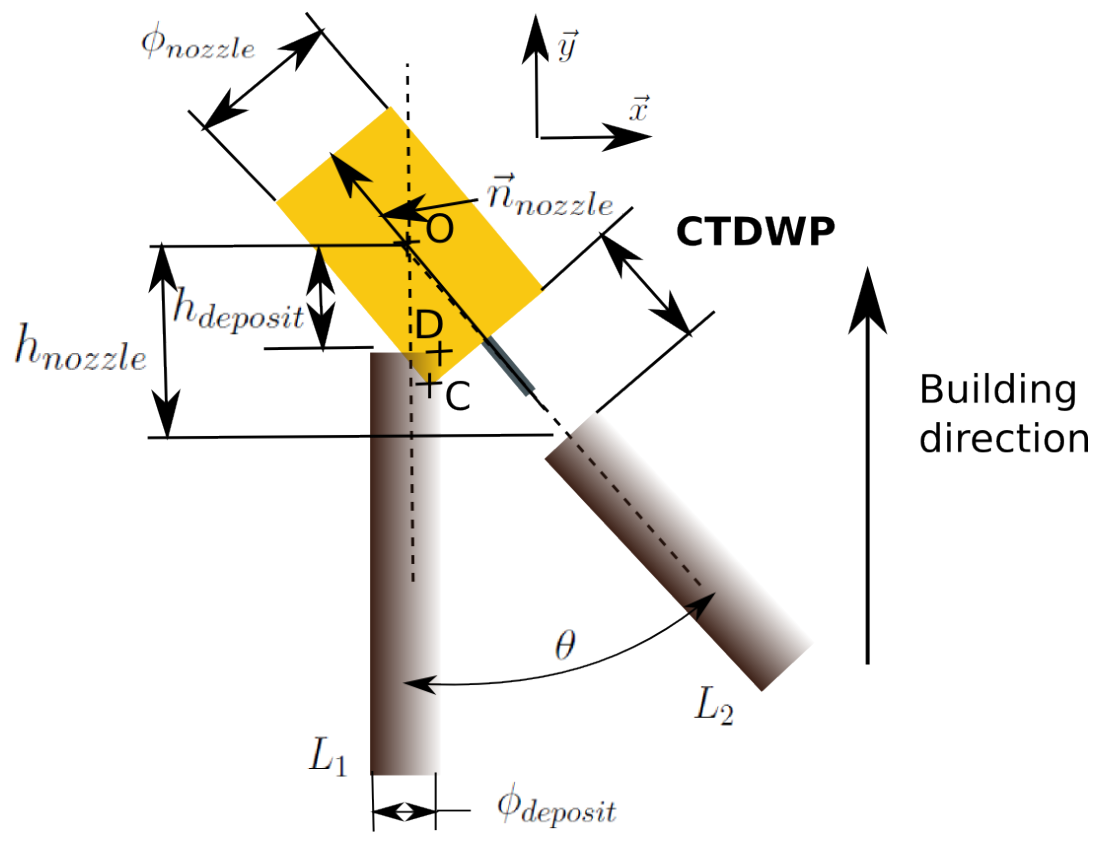

Figure 6: A possible collision between the tool (nozzle) and the left vertical deposit polyline. The point $\mathrm{D}$ belongs to the deposit and point $\mathrm{C}$ to the nozzle. The direction of the tool is $\vec{n}_{n o z z l e}$ and the direction of the line $L_{1}$ is $\vec{n}_{\text {deposit }}$.

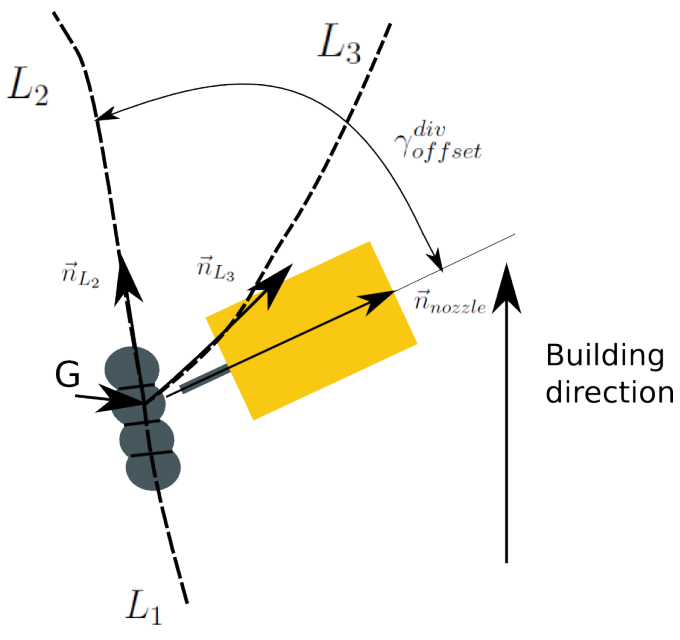

(a)

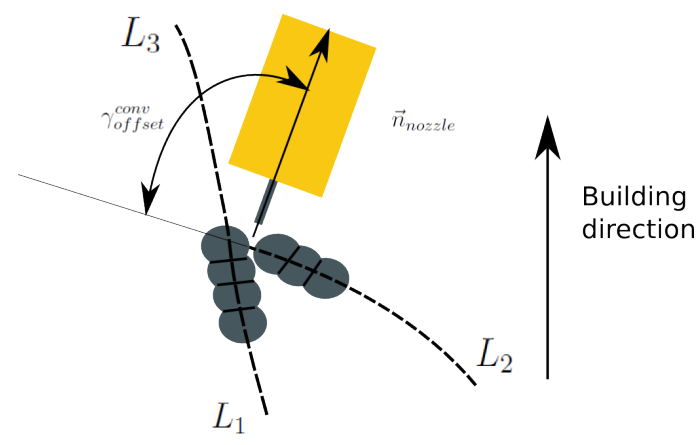

(b)

Figure 7: Different types of intersections relative to the building direction. Right: divergent case, Left: convergent intersection. The nozzle is represented with the chosen strategy. 




Figure 8: Overall algorithm for the slicer, the manager and the control of SAMM Process. 


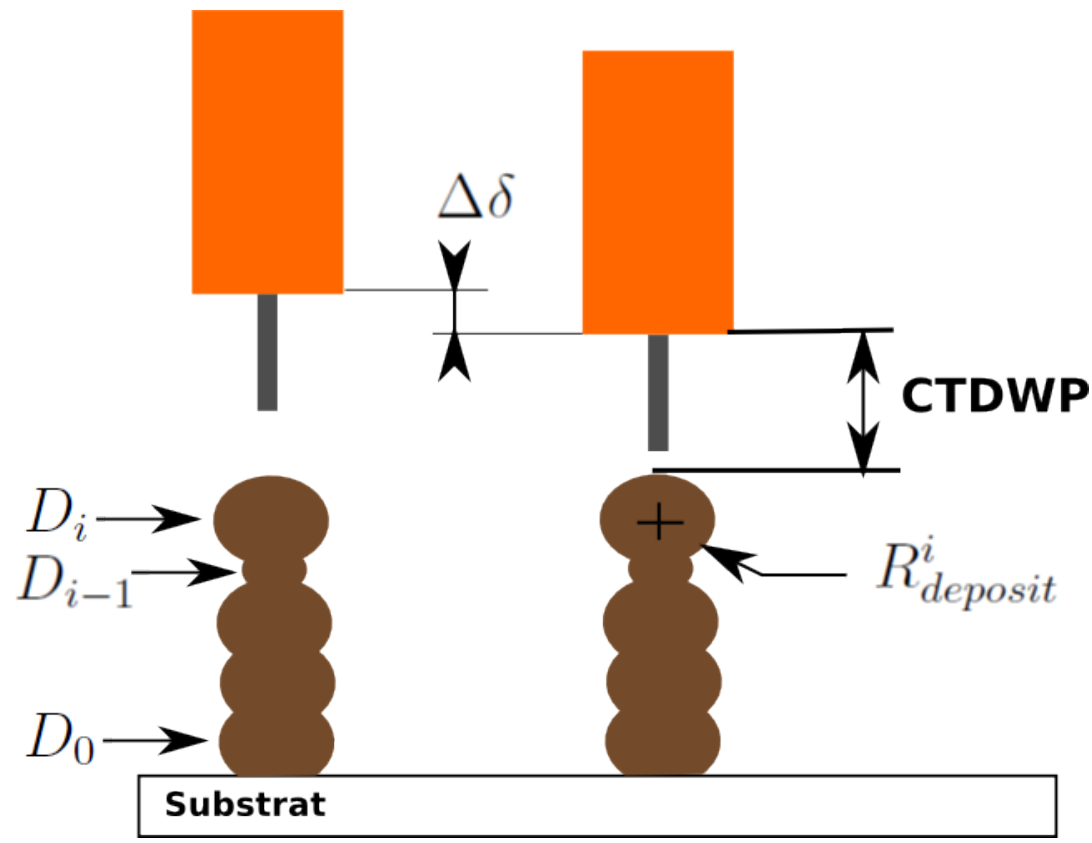

Figure 9: Linear vertical deposition without (left) and with (right) control.
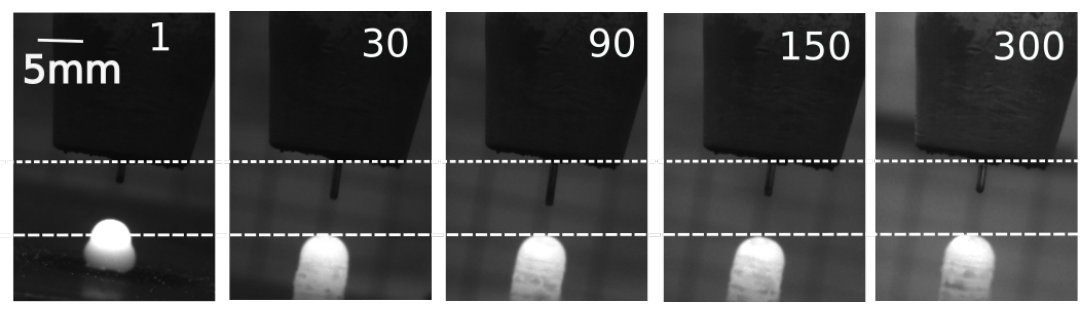

Figure 10: A series of images taken during the building of a vertical line. The number corresponds to the built point. 


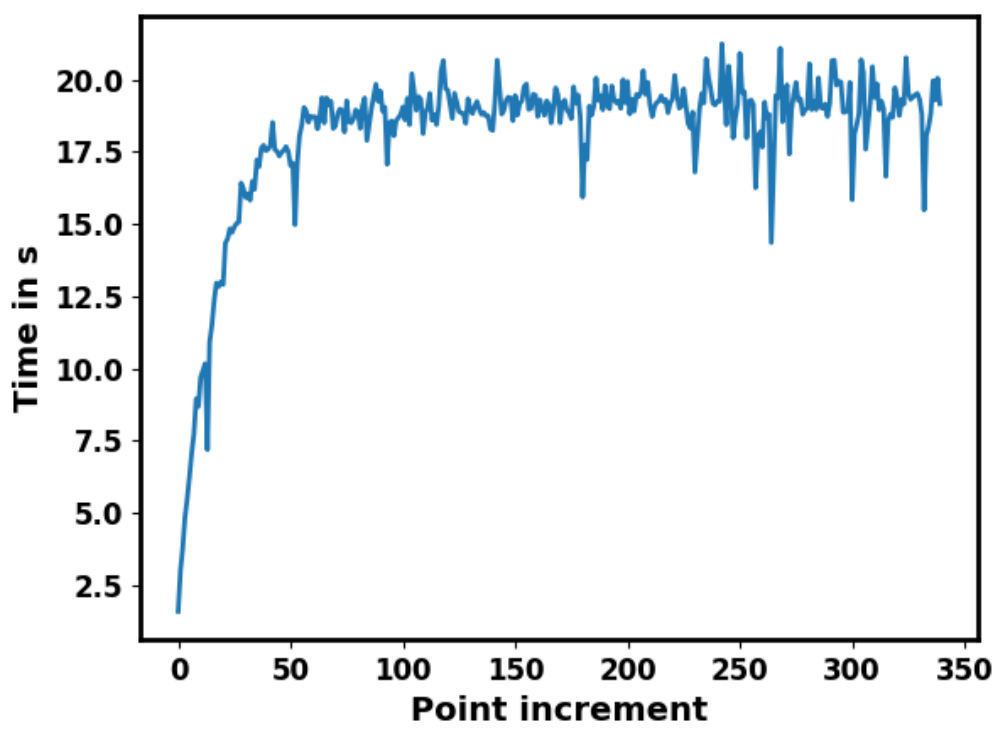

Figure 11: Time to obtain the image in function of the point increment during vertical line manufacturing. 


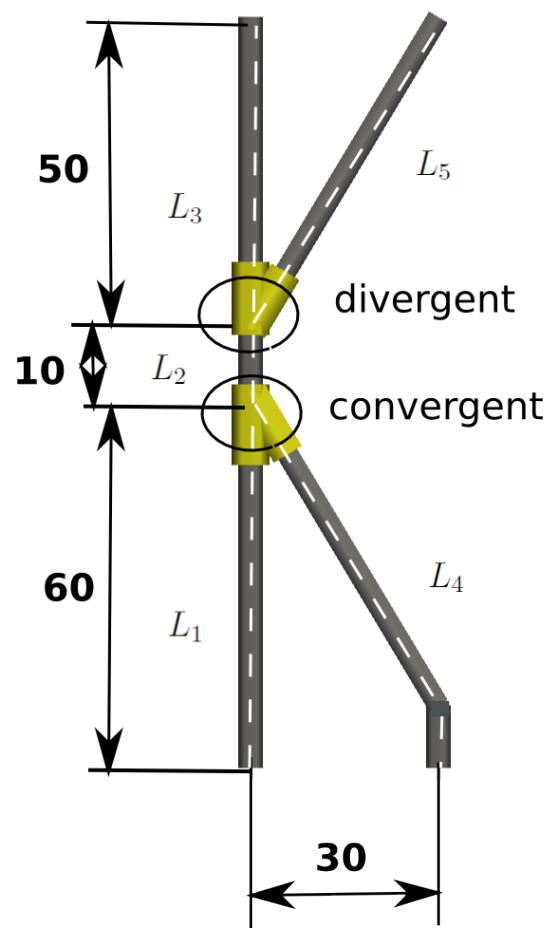

Figure 12: The geometry defined in the CAM part and the zone of conflicts during deposition process. 


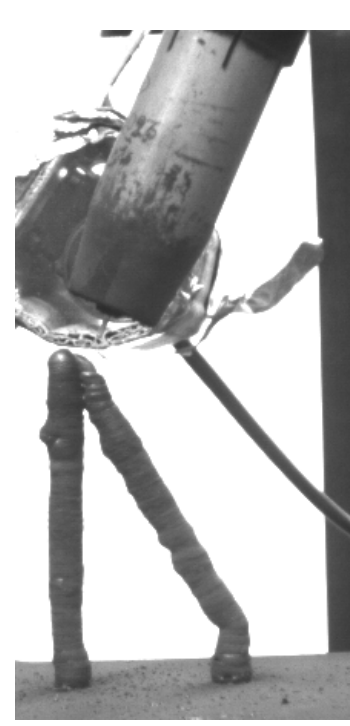

(a)

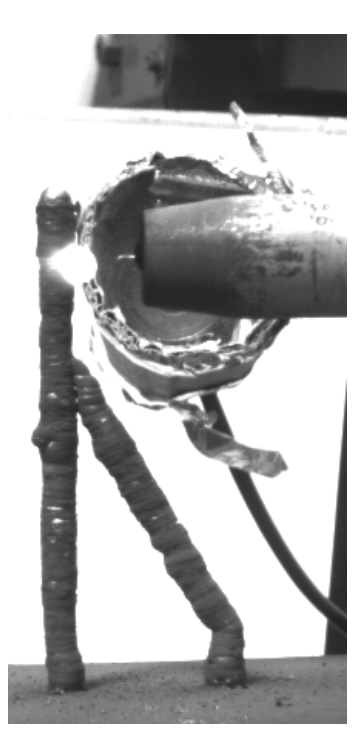

(b)

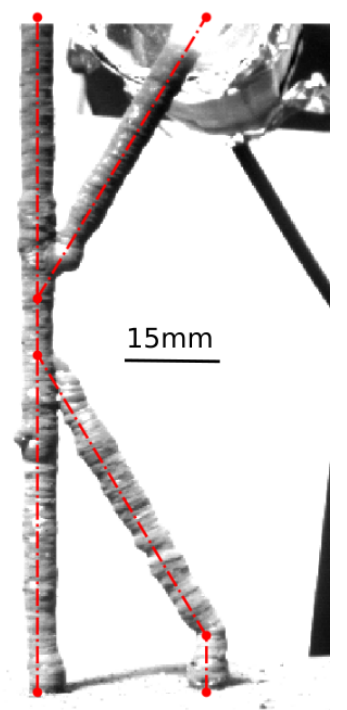

(c)

Figure 13: Intersection manufacturing strategies and final free-form shape. Monitoring camera can be seen behind the torch. 


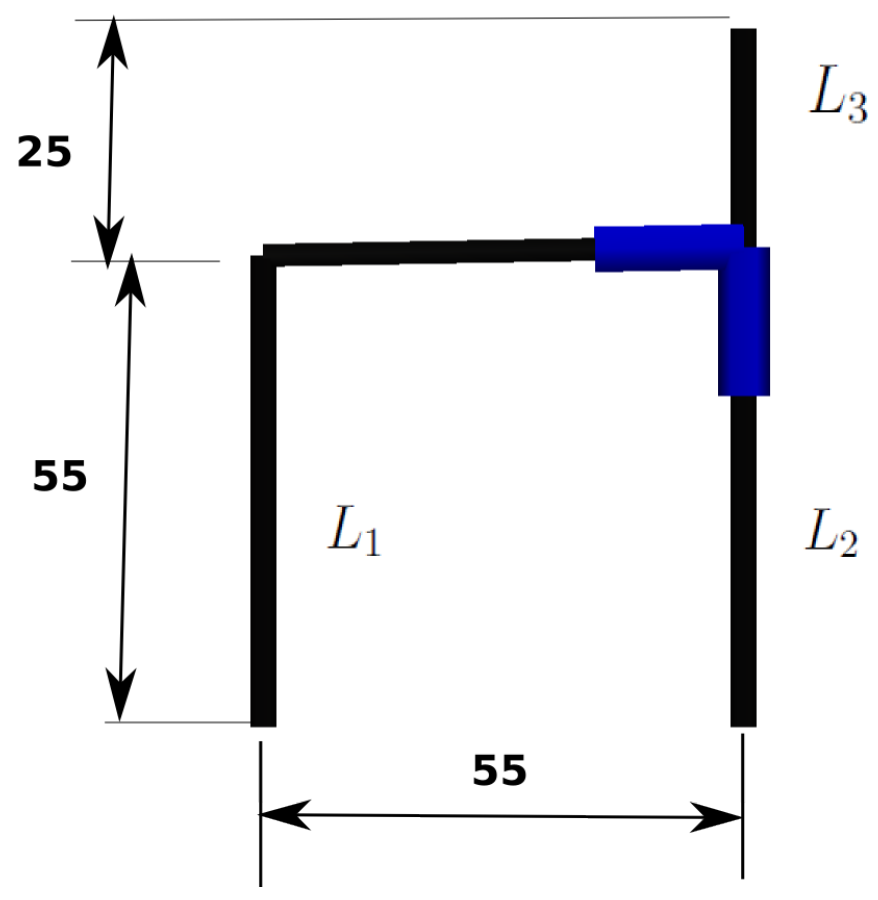

Figure 14: The geometry defined in the CAM part and the zone of collisions during the deposition process.

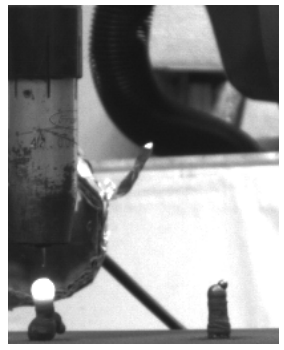

(a)

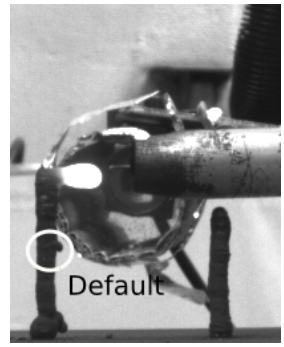

(b)

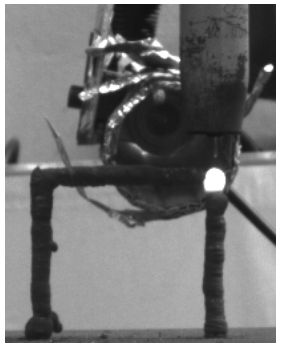

(c)

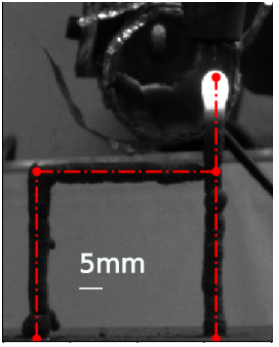

(d)

Figure 15: Evolving geometry during the manufacturing of the chair. 

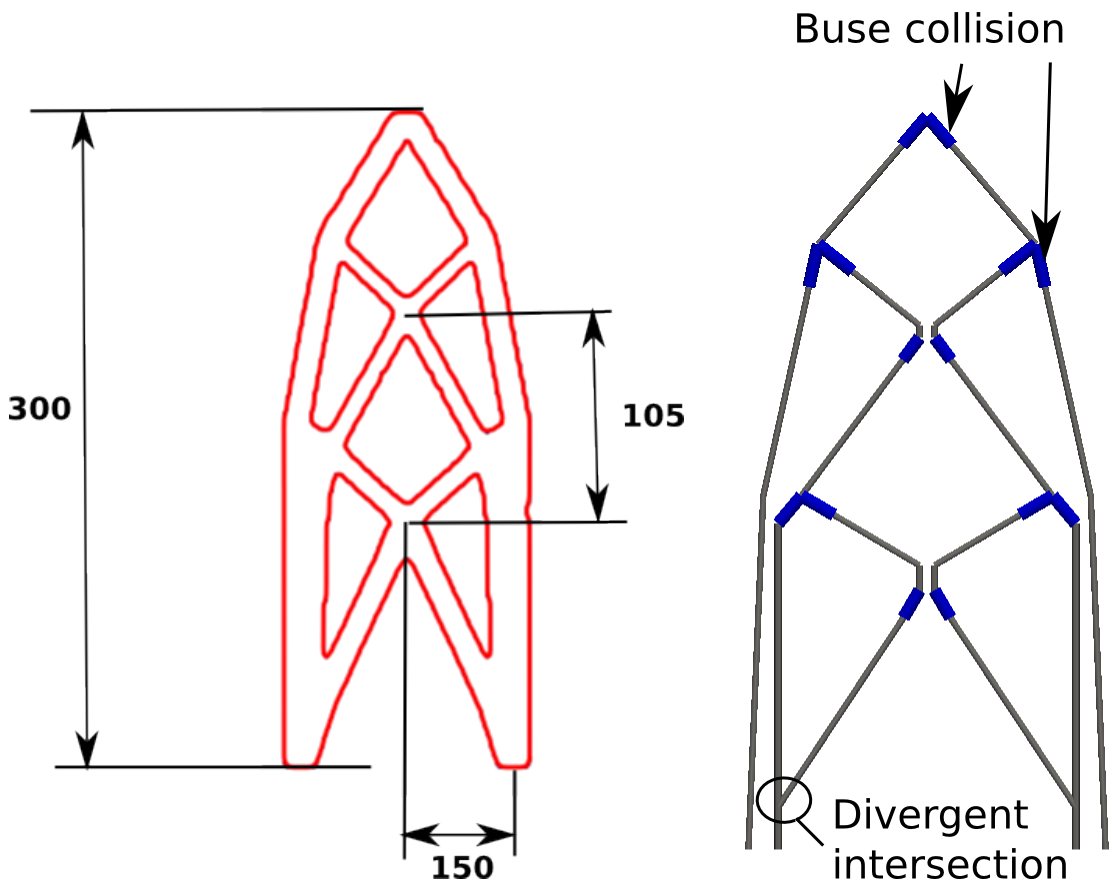

Figure 16: Geometry obtained by shape optimization for a cantilever beam loaded by a transversal force at the top of the structure. The right figure corresponds to the conversion of the geometry into a geometry for point by point.

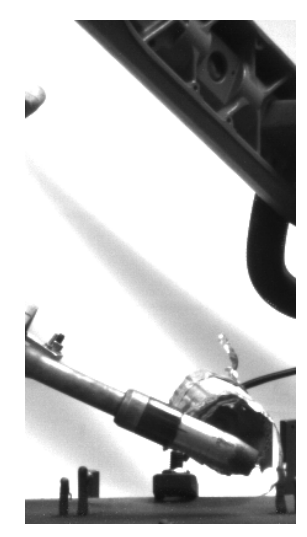

(a)

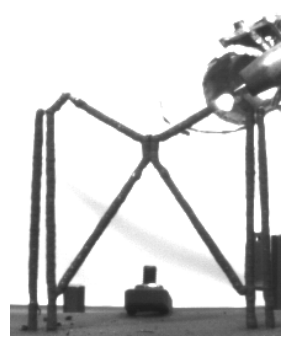

(b)

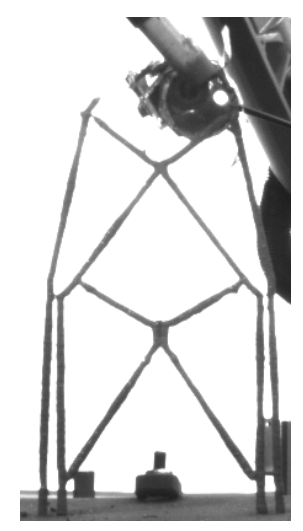

(c)

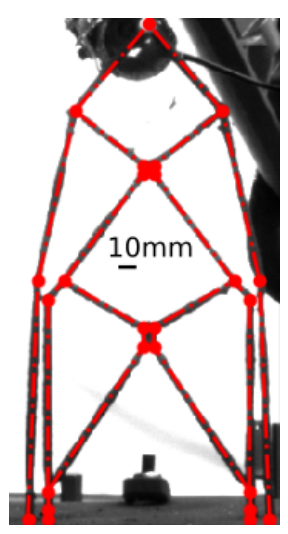

(d)

Figure 17: Evolving geometry during the manufacturing of the cantilever beam. 


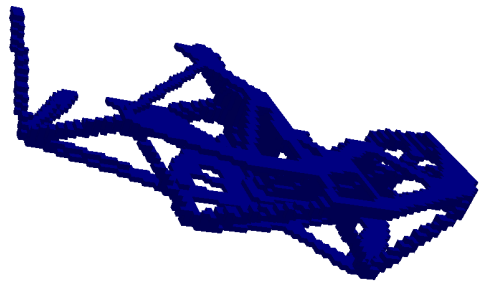

(a)

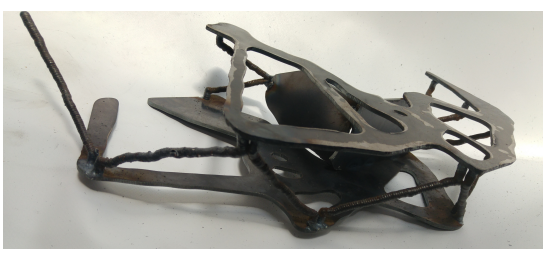

(b)

Figure 18: Evolving geometry during the manufacturing of a goose neck structure. 